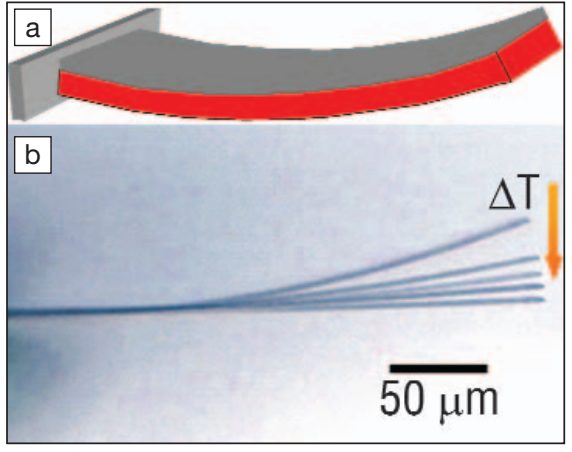

Figure 1. (a) Schematic illustration of a bimaterial cantilever bending with incident heat. (b) Side view of an optical image of a polymer-silicon microcantilever bending as the temperature increases $20-40^{\circ} \mathrm{C}$. Reproduced with permission from Nano Letters 6 (4) (April 12, 2006) p. 730; DOI: 10.1021/nl0525305. (C) 2006 American Chemical Society.

sometry to be in the range of $20-200 \mathrm{~nm}$. AFM revealed that the PS layer has a smooth, uniform surface morphology and an elastic modulus of nearly $2 \mathrm{GPa}$. In addition, the PS could not be dissolved in or swollen by organic solvents. Compressive residual stresses arising from PECVD of PS result in a bent and stressed bimorph beam (see Figure 1), whose initial parameters can be controlled by deposition conditions.

The researchers showed that deflections of their microcantilever as a function of temperature within either a narrow temperature range $\left(30-31^{\circ} \mathrm{C}\right)$ or a broader range $\left(20-45^{\circ} \mathrm{C}\right)$ are many times higher than those of a reference microcantilever composed of silicon with a 60-nm gold layer, which is the common materials platform for uncooled IR bimaterial microsensors. The thermal sensitivity of $1-2 \mathrm{~nm} / \mathrm{mK}$ is much higher than that displayed by the gold-silicon reference $(0.056 \mathrm{~nm} / \mathrm{mK})$ or the value achieved by the best uncooled microcantilever IR detectors $(0.12 \mathrm{~nm} / \mathrm{mK})$ currently on the market. Using a mathematical analysis, the researchers concluded that the intrinsic stress within the PS layer reverses sign and becomes tensile at the elevated temperature at which their microcantilevers attain planarity. In a temperature range of $20-45^{\circ} \mathrm{C}$, the overall variability between the first and 100th cycle was $<1.5 \%$, and the thermal sensitivity varies only about $1 \%$. The researchers said, "Due to the wide variety of monomers that can be plasmadeposited, our approach allows for further chemical modification to make multifunctional chemical-thermal micro- sensor arrays with tunable spectral response" and the dry polymer deposition process "is compatible with batch microfabrication processing."

STEVEN TROHALAKI

\section{DNA and Gold Nanoparticles Form 3D Nanoscaffolds}

Programmed nanostructures, ordered two- and three-dimensional nanoparticle arrays, nanoelectronics, nanophotonics, biosensing, bioimaging, and biodiagnostics are some of the potential applications for DNA-nanoparticle bioconjugates. DNA's fabrication advantages include its physicochemical stability, mechanical rigidity, specific base-pairing, and predictable intermolecular and intramolecular interactions. In addition, mature enzymatic methods exist for DNA modification as well as for DNA amplification-the production of many DNA copies from one or a few originals. Rolling circle amplification (RCA) is one powerful variation in which a DNA polymerase continuously grows a DNA chain by adding nucleotides according to a circular DNA template. Recently, M.A. Brook, Y. Li, and co-researchers of McMaster University have used RCA to construct unique 3D scaffolds composed of DNA and gold nanoparticles (Au NPs).

As described in the April 3 issue of Angewandte Chemie International Edition (p. 2409; DOI: 10.1002/anie.200600061), the researchers used an established method to attach thiol-modified singlestranded (ss) DNA primers, 41 nucleotides in length, to gold nanoparticles, $15 \mathrm{~nm}$ in diameter. UV-vis spectroscopy showed that each structure (called primer-Au) had about 230 ssDNA primer strands, on average. A complementary 63-nucleotide circular, ssDNA template was then annealed to the primer-Au with $\sim 30 \%$ efficiency. Under conditions that the researchers determined to be optimal, RCA was performed with the DNA polymerase $\phi 29 D N A P$ at a relatively low primer-Au concentration $(\sim 0.67 \mathrm{nM})$ for a relatively short time (30 $\mathrm{min})$ to produce structures (termed long-DNA $\mathrm{Au}$ ) composed of $\mathrm{Au}$ NPs with long ssDNA attached (see Figure 1). The researchers used gel electrophoresis, before and after digestion with restriction enzymes, and atomic force microscopy (AFM) to show that RCA produced high-molecular-weight DNA in excess of 1000 nucleotides.

The long-DNA Au structures were then assembled into 3D scaffolds by overnight incubation with 5-nm Au NPs modified with 25-nucleotide complementary oligonucleotides that form Watson-Crick base pairs - that is, double-stranded DNA (dsDNA) - with the long ssDNA (see
Figure 1). The resulting complexes were isolated using low-speed centrifugation. Transmission electron microscopy showed that the 5-nm Au NPs reside in the area surrounding the 15-nm Au NPs (see Figure 2). The distance between the 5-nm

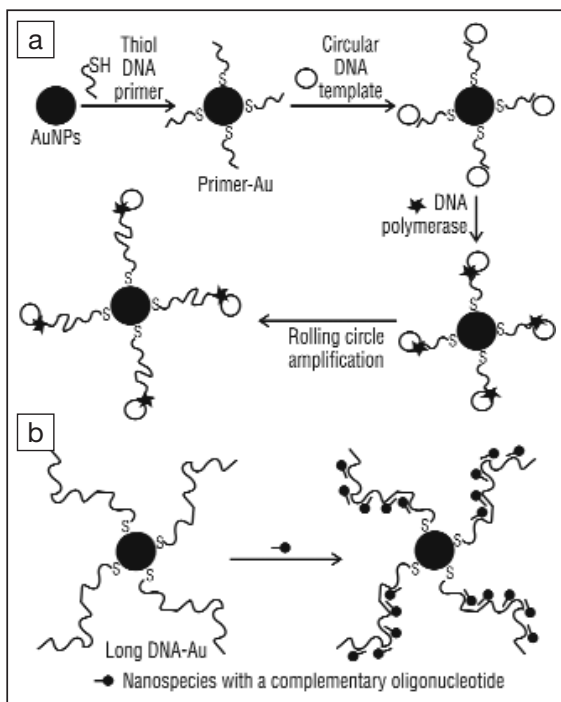

Figure 1. (a) Schematic illustration of rolling circle amplification (RCA) on gold nanoparticles (Au NPs). (b) DNA-Au conjugates produced by RCA as a scaffold for the formation of three-dimensional nanostructures. Reproduced with permission from Angewandte Chemie International Edition 45 (15) (2006), p. 2409; DOI: 10.1002/anie.200600061. (C) 2006 Wiley-VCH, Weinheim.

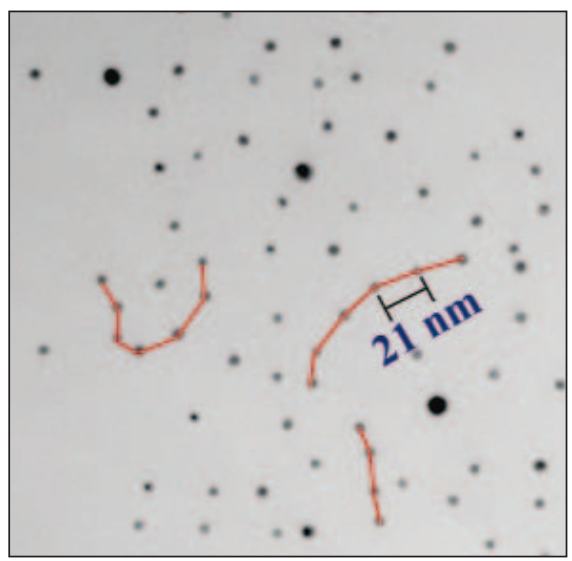

Figure 2. Transmission electron micrograph of nanoassembled superstructures prepared from 5-nm gold nanoparticles modified with antisense DNA and longDNA Au scaffolds. Reproduced with permission from Angewandte Chemie International Edition 45 (15) (2006) p. 2409; DOI: 10.1002/anie.200600061. (C) 2006 Wiley-VCH, Weinheim. 


\section{Measure the Thermoelectric Effect with the Seebeck Coefficient Measuring System}

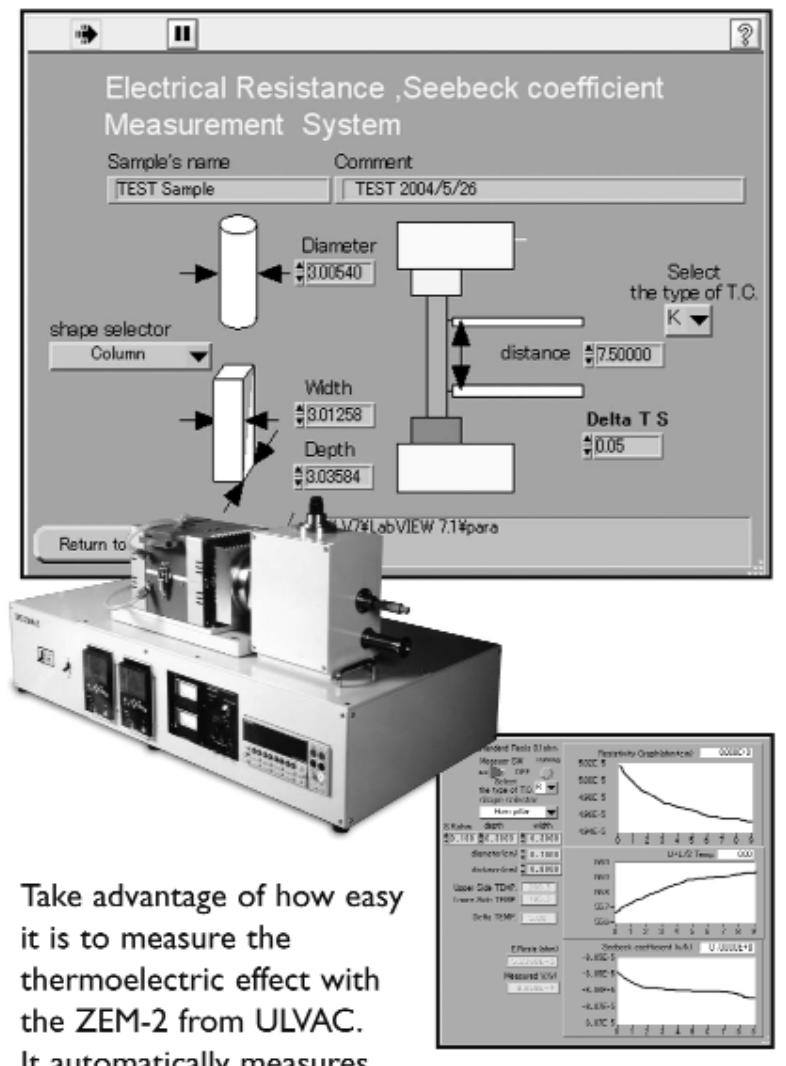

It automatically measures

both the Seebeck Coefficient and resistance of bulk samples, thin sheet or thin film deposited thermoelectric materials.

The bench top, computer-controlled ZEM-2 features:

- Fully automatic operation

- Operating range from -80 to $1,000^{\circ} \mathrm{C}$

- Easy sample loading and pumpdown

Just enter your sample geometry, experimental parameters and data reporting preferences and the ZEM-2 does the rest. For the best in productivity and accuracy, choose the ZEM-2 system.

With ZEM-2 Just run the samples!

\section{ULVAC}

ULVAC Technologies, Inc Methuen, MA 01844

Phone: 978-686-7550

www.ulvac.com

sales@ulvac.com
Au NPs measured along the long-DNA is $\sim 21 \mathrm{~nm}$, which is consistent with the length of the 63-nucleotide circular DNA template and the distance between base pairs in the B form of dsDNA $(0.34$ $\mathrm{nm})$. This data, as well as AFM studies, strongly suggested to the researchers that the nanoassembly was well defined and periodic. The researchers said that the drawbacks of their system, including polydispersity associated with RCA, are far outweighed by the advantages: the periodicity in 3D is well defined; the distance between the assembled nanospecies can be readily controlled; the assembly is entirely reversible; and multiple assemblies, wherein two or more nanospecies can be simultaneously constructed, can be easily realized.

STEVEN TROHALAKI

\section{Mixed Hydride Material Offers Hydrogen Storage Solutions}

The major roadblock to moving toward a hydrogen economy involves issues with the storage of hydrogen, especially on vehicles. Despite promising strides made in the development of hydrogen storage materials, new and more complex materials systems still need to be developed to adequately resolve the challenges associated with storing hydrogen. In the May issue of Chemical Communications (DOI: 10.1039/b518243c), a team of researchers led by P.A. Anderson from the University of Birmingham (UK), W.I.F. David from the Rutherford Appleton Laboratory (Didcot, UK), and P.P. Edwards from the University of Oxford (UK), have reported the synthesis and structure of a complex hydride formed by combining two potential hydrogen storage materials, $\mathrm{LiNH}_{2}$ and $\mathrm{LiBH}_{4}$.

The novel mixed hydride material has a nominal composition of $\mathrm{Li}_{4} \mathrm{BN}_{3} \mathrm{H}_{10}$, but accommodates a wide range of stoichiometries. The researchers were able to use infrared spectroscopy to determine that the $\mathrm{BH}_{4}-$ and $\mathrm{NH}_{2}$ - ions remain intact in their material, demonstrating, they said, that the structure is best regarded as $\mathrm{Li}_{4} \mathrm{BH}_{4}\left(\mathrm{NH}_{2}\right)_{3}$. The structure was determined from powder diffraction data using high-intensity x-rays generated by a synchrotron and from neutron powder diffraction experiments. The $\mathrm{B}-\mathrm{H}$ bonds in the solid solution appear to be stronger relative to the $\mathrm{LiBH}_{4}$ starting material, but one of the $\mathrm{B}-\mathrm{H}$ bonds is significantly shorter than the other three. In contrast, the $\mathrm{N}-\mathrm{H}$ bonds seem to be weaker than in $\mathrm{LiNH}_{2}$, as reported by the research team.

Most remarkably, the researchers said, this mixed borohydride amide, with a structure similar to $\mathrm{LiNH}_{2}$, shows a different decomposition behavior, almost exclusively evolving hydrogen instead of ammonia. The researchers said that this behavior may originate from the molecular proximity of positively charged hydrogen atoms in $\mathrm{NH}_{2}$ and negatively charged hydrogen in the $\mathrm{BH}_{4}$ - anions. Anderson said, "The ability to modify chemically the decomposition pathway of hydrogen-containing compounds could lead to a whole range of completely new hydrogen storage candidates."

SARBAJIT BANERJEE

\section{Magneto-Optical Surface Plasmon Resonance Sensor Enhances Detection of Biomolecules}

Surface plasmons are collective excitations of electrons at the interface between a conductor and a dielectric material. Surface plasmon resonance (SPR) is a strong function of the refractive index of the insulator. Conventional SPR-based biosensors are able to resolve differences in refractive index of $\sim 10^{-5}$, which corresponds to a detection sensitivity of $1-5 \mathrm{pg} / \mathrm{mm}^{2}$ of biomolecules adsorbed at the sensor surface. For direct detection of small molecules, resolutions approaching $0.1 \mathrm{pg} / \mathrm{mm}^{2}$ or lower are required. In the April 15 issue of Optics Letters (p. 1085), B. 